\title{
Correction to: Low-level laser irradiation at a high power intensity increased human endothelial cell exosome secretion via Wnt signaling
}

\author{
Hesam Saghaei Bagheri ${ }^{1}$ - Monireh Mousavi ${ }^{2}$. Aysa Rezabakhsh ${ }^{1}$ - Jafar Rezaie ${ }^{1}$ Seyed Hossein Rasta ${ }^{3,4}$. \\ Alireza Nourazarian ${ }^{5} \cdot$ Çigir Biray Avci ${ }^{6} \cdot$ Habib Tajalli $^{7} \cdot$ Mehdi Talebi $^{8} \cdot$ Ahmad Oryan $^{9} \cdot$ Majid Khaksar $^{1}$. \\ Masoumeh Kazemi ${ }^{1}$ - Seyed Mahdi Nassiri ${ }^{10}$. Shahrooz Ghaderi ${ }^{11}$ • Bakiye Goker Bagca ${ }^{6}$. Reza Rahbarghazi ${ }^{1,12}$. \\ Emel Sokullu ${ }^{13,14}$
}

Published online: 28 November 2019

C Springer-Verlag London Ltd., part of Springer Nature 2019

\section{Correction to: Lasers in Medical Science.} https://doi.org/10.1007/s10103-018-2495-8

After publication of this paper, the authors determined an error in Fig. 4. Below is the correct Fig. 4.

The online version of the original article can be found at https://doi.org/ $10.1007 / \mathrm{s} 10103-018-2495-8$

Reza Rahbarghazi

rezarahbardvm@gmail.com; rahbarghazir@tbzmed.ac.ir

$\triangle$ Emel Sokullu

emelsu@gmail.com; emel.sokullu@ikc.edu.tr

1 Stem Cell Research Center, Tabriz University of Medical Sciences, Imam Reza St., Golgasht St, Tabriz 5166614756, Iran

2 Department of Genetic, Islamic Azad University, Ahar Branch, Ahar, Iran

3 Medical Physics Department, Medical Faculty, Tabriz University of Medical Sciences, Tabriz, Iran

4 School of Medical Sciences, University of Aberdeen, Aberdeen, UK

5 Department of Biochemistry and Clinical Laboratories, Faculty of Medicine, Tabriz University of Medical Sciences, Tabriz, Iran

6 Department of Medical Biology, Faculty of Medicine, Ege University, Izmir, Turkey
7 Research Institute for Applied Physics and Astronomy, University of Tabriz, Tabriz, Iran

8 Hematology and Oncology Research Center, Tabriz University of Medical Sciences, Tabriz, Iran

9 Department of Pathobiology, Faculty of Veterinary Medicine, Shiraz University, Shiraz, Iran

10 Department of Clinical Pathology, Faculty of Veterinary Medicine, University of Tehran, Tehran, Iran

11 Department of Molecular Medicine, Faculty of Advanced Medical Sciences, Tabriz University of Medical Sciences, Tabriz, Iran

12 Department of Applied Cell Sciences, Faculty of Advanced Medical Sciences, Tabriz University of Medical Sciences, Tabriz, Iran

13 Bioengineering Department, Izmir Katip Celebi University, 35100 Izmir, Turkey

14 Division of Biomedical Engineering at Brigham and Women's Hospital, Harvard-MIT Health Sciences and Technology, Harvard Medical School, Cambridge, MA, USA 
Fig. 4 Autophagy status of HUVECs after exposure to LLLI (a). Laser irradiation could promote autophagy response by increasing LC3-II/I and reducing P62 levels in HUVECs at a dose of $80 \mathrm{~J} / \mathrm{cm}^{2}$. Measurement of mitochondrial membrane potential $(\Delta \Psi)$ using Rhodamine 123 (b). Real-time PCR analysis of Optineurin and PINK1 (c).

Data showed the normal expression of these genes during LLLI. One-way ANOVA with Tukey's post hoc test. $* * p<0.01$; $* * * p<0.001 ; * * * * p<0.0001$ a

P62

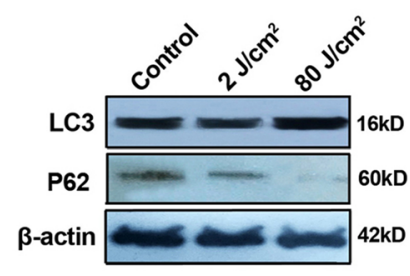

b

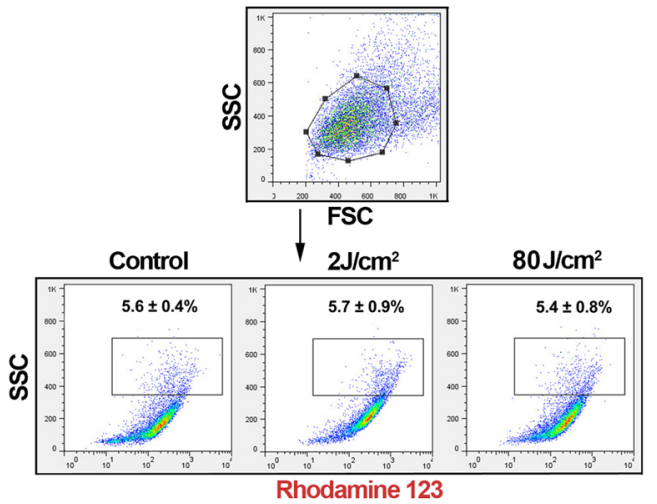

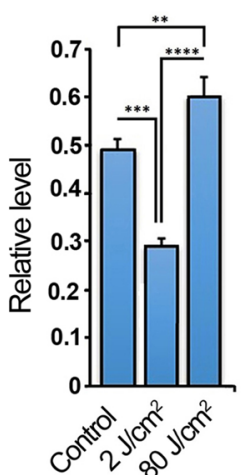

Rhodamine 123 staining

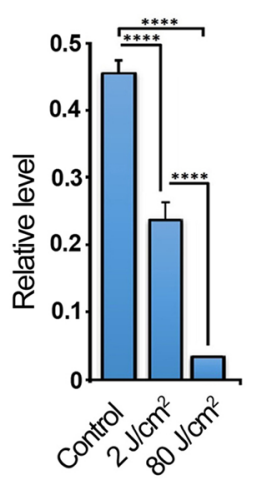

C
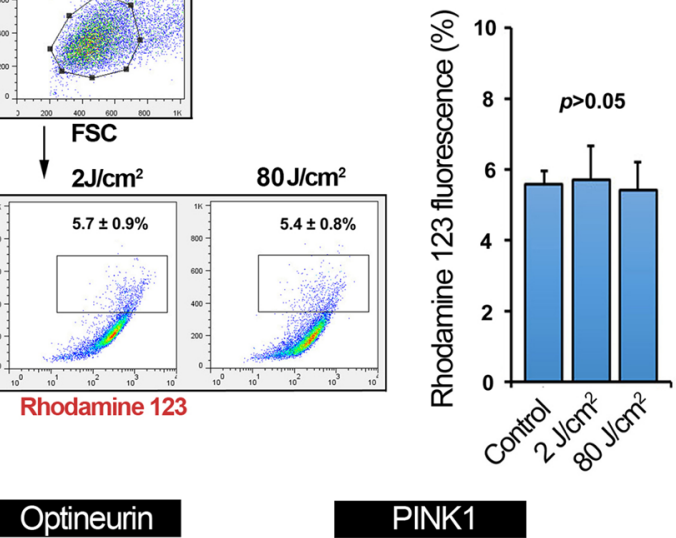

PINK1
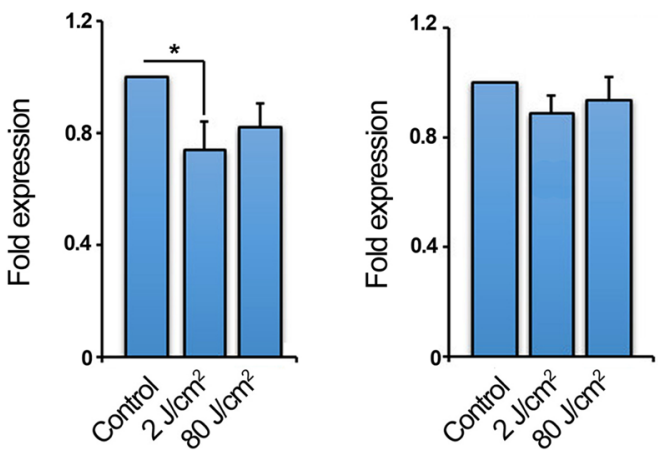

Publisher's note Springer Nature remains neutral with regard to jurisdictional claims in published maps and institutional affiliations. 